\title{
CRYSTAL STRUCTURES OF LARGE-VOLUME COMMERCIAL PHARMACEUTICALS
}

\author{
James A. Kaduk ${ }^{1}$, Ryan L. Hodge ${ }^{2}$, Nicholas C. Boaz ${ }^{2}$, Amy M. Gindhart ${ }^{3}$, Thomas N. Blanton ${ }^{3}$ \\ ${ }^{I}$ North Central College, Naperville IL, Illinois Institute of Technology, Chicago IL Poly Crystallography Inc., Naperville IL \\ ${ }^{2}$ North Central College, Naperville IL \\ ${ }^{3} I C D D$, Newtown Square PA \\ kaduk@polycrystallography.com
}

As part of a continuing project, the challenging room-temperature crystal structures of eight commercial pharmaceutical APIs have been solved by Monte Carlo simulated annealing techniques using synchrotron X-ray powder diffraction data (11-BM at APS), and optimized using density functional techniques. Tofacitinib dihydrogen citrate (Xeljanz $\left.{ }^{\circledR}\right),\left(\mathrm{C}_{15} \mathrm{H}_{21} \mathrm{~N}_{6} \mathrm{O}\right)\left(\mathrm{H}_{2} \mathrm{C}_{6} \mathrm{H}_{5} \mathrm{O}_{7}\right)$, crystallizes in $P 2{ }_{1}{ }_{2}{ }_{1}{ }_{1}$ with $a=5.91113(1), b=12.93131(3), c=30.43499(7) \AA, V=2326.411(6) \AA^{3}$, and $Z=4$. All of the "interesting" hydrogn atoms could be located by analysis of potential hydrogen bonding patterns. Eltrombopag olamine Form I (Promactå), $\left(\mathrm{C}_{2} \mathrm{H}_{8} \mathrm{NO}\right)_{2}\left(\mathrm{C}_{25} \mathrm{H}_{20} \mathrm{~N}_{4} \mathrm{O}_{4}\right)$ crystallizes in $P 2_{1} / n$ with $a=17.65884(13), b=7.55980(2), c=22.02908(16) \AA, \hat{a}=105.8749(4) \mathrm{E}, V=$ 2828.665(11) $\AA^{3}$, and $Z=4$. The initial structure solution reversed the orientation of one of the cations. Levocetirizine hydrochloride Form I (Zyzal), $\mathrm{C}_{21} \mathrm{H}_{27} \mathrm{ClN}_{2} \mathrm{O}_{3} \mathrm{Cl}$, apparently crystallizes in $P 2_{1} / n$ (even though it is a chiral molecule and exhibits weak second-harmonic generation) with $a=24.1318(21), b=7.07606(9), c=13.5205(7), \hat{a}=97.9803(4) \mathrm{E}, V=2286.38(12) \AA^{3}$, and $Z$ $=4$.

Edoxaban tosylate monohydrate Form I (Lixiana $\left.{ }^{\circledR}\right),\left(\mathrm{C}_{24} \mathrm{H}_{31} \mathrm{ClN}_{7} \mathrm{O}_{4} \mathrm{~S}\right)\left(\mathrm{C}_{7} \mathrm{H}_{7} \mathrm{O}_{3} \mathrm{~S}\right)\left(\mathrm{H}_{2} \mathrm{O}\right)$, crystallizes in $P 2_{1}$ with $a=7.55097(2), b$ $=7.09010(2), c=32.08420(21) \AA, \hat{a}=96.6720(3) \mathrm{E}, V=1744.348(6) \AA^{3}$, and $Z=2$. Tezacaftor Form A (Symdeko), $\mathrm{C}_{26} \mathrm{H}_{27} \mathrm{~F}_{3} \mathrm{~N}_{2} \mathrm{O}_{6}$, crystallizes in $C 2$ with $a=21.05142(2), b=6.60851(2), c=17.76032(5) \AA, \hat{a}=95.8255(2) \mathrm{E}, V=2458.027(7) \AA^{3}$, and $Z=4$. Pomalidomide Form I (Pomalyst), $\mathrm{C}_{13} \mathrm{H}_{11} \mathrm{~N}_{3} \mathrm{O}_{4}$, crystallizes in $P-1$ with $a=7.04742(9), b=7.89103(27), c=11.3106(6) \AA, a=$ 73.2499(13), $\hat{a}=80.9198(9), \tilde{a}=88.5969(6) \mathrm{E}, V=594.618(8) \AA^{3}$, and $Z=2$. Palbociclib isethionate Form B (Ibrance ${ }^{\circledR}$ ), $\left(\mathrm{C}_{24} \mathrm{H}_{30} \mathrm{~N}_{7} \mathrm{O}_{2}\right)\left(\mathrm{C}_{2} \mathrm{H}_{5} \mathrm{O}_{4} \mathrm{~S}\right)$, crystallizes in $P-1$ with $a=8.71337(4), b=9.32120(6), c=17.73722(20) \AA$, $a=80.0258(5), \hat{a}=$ 82.3581(3), $\tilde{a}=76.1560(2) \mathrm{E}, V=1371.284(5) \AA^{3}$, and $Z=2$. Osimertinib mesylate Form B (Tagrisso), $\left(\mathrm{C}_{28} \mathrm{H}_{34} \mathrm{~N}_{7} \mathrm{O}_{2}\right)\left(\mathrm{CH}_{3} \mathrm{O}_{3} \mathrm{~S}\right)$ crystallizes in $P-1$ with $a=11.4291(3), b=11.7223(4), c=13.3221(4), \dot{a}=69.0246(8), \hat{a}=74.5906(7), \tilde{a}=66.4001(7) \mathrm{E}, V=$ $1511.466(13) \AA^{3}$, and $Z=2$. Other new structures may be discussed as they become available.

Keywords: pharmaceutical, powder diffraction, Rietveld refinement, density functional theory 